\title{
ÉTUdE DE LA PRODUCTION DE PRÉESTIVAUX DE CORÉGONE (COREGONUS sp.) EN CAGES ÉCLAIRÉES IMMERGEESS DANS LE LEMAN
}

\author{
A. Champigneulle *, M. MiChoud * , L. DUCRet **, G. fOUSSAT **, \\ H. MUDRY **, D. NOEL ** et G. ZEGNA **
}

\footnotetext{
* Station d'Hydrobiologie Lacustre, INRA, B.P. 11 F, 75 avenue de Corzent, 74203 THONON-lesBAINS, France.

** Pisciculture de Rives, D.D.A. de Haute-Savoie, quai de Rives, 74200 THONON-les-BAINS, France.

*** Conseil Supérieur de la Pêche et Fédération des A.P.P. de Haute-Savoie.
}

\begin{abstract}
RESUME
Les résultats des récents essais (1983 à 1985) français d’élevage de préestivaux de corégones en cages éclairées immergées dans le Léman sont présentés. Les fluctuations interannuelles de la production sont fortes. La période de mise en charge, en liaison avec les conditions thermiques et trophiques, influe sur la croissance et la survie au cours des premières semaines. L'utilisation de la technique d'élevage en cages est discutée et des recommandations sont faites.
\end{abstract}

\section{SUMMARY}

EVALUTATION OF THE RESULTS OF AN EXPERIMENT OF REARING COREGONID (COREGONUS SP.) FRY IN ILLUMINATED CAGES SUBMERGED IN LAKE LEMAN.

The paper presents the results of a recent three-years french experiment of rearing coregonid /Coregonus sp./ fry in illu minated cages submerged in Lake Leman. The date of stocking in connection with the thermic and trophic conditions are determinant for the growth and survival during the first weeks. The use of the technique of rearing in cages is discussed and some recommendations are presented.

\section{INTRODUCTION}

En France les corégones sont bien représentés dans les grands lacs subalpins (Léman, Annecy et Bourget) et dans les lacs du Jura. Une diminution des captures est observée dans les lacs du Bourget et le Léman qui sont en cours d'eutrophisation. Dans le Bourget les captures ont fortement diminué depuis 20 ans. Dans le Léman les prises de corégones ont fluctué entre 50 et 220 t. durant 27 années consécutives (1950-76). Mais les captures ont été inférieures à $60 \mathrm{t}$. pour chacune des 9 dernières années sauf 1978. En France, de même qu'en Amérique du Nord et en Europe, des travaux récents visent à connaître et à améliorer l'efficacité des repeuplements en corégones (LUCZYNSKI, 1984 a et b; PRUUKI et al., 1984 ; WESTMAN et al., 1984 ; GERDEAUX et DEWAELE, 1986; MENG et al., 1986 TODD, 1986).

Plusieurs études (VAN OSTEN, 1942; MILLER, 1946; BATIAS, 1954; QUARTIER, 1954; CHRISTIE, 1963 ; NÜHMANN, 1972 et TODD, 1986) ont montré que les relâchers au stade "alevins vésiculés" sont peu efficaces dans les grands lacs où il existe déjà un stock en place dont la reproduction naturelle est efficace. L'efficacité des relâchers d'alevins vésiculés semble meilleure dans les petits lacs avec peu de prédateurs et sans corégones en place (CHAMPIGNEULLE, 1985) ou lorsque le relâcher peut être retardé par incubation des œufs en eau froide (NÜHMANN, 1972 et LUCZYNSKI, 1984b) ou à partir d'un seuil minimal de mise en charge (GERDEAUX et DEWAELE, 1986 et TODD. 1986).

Certains auteurs (EINSELE, 1965 ; VOLGIN, 1973 ; PRUKI et al., 1984 ; WESTMAN et al., 1984 ; LUCZYNSKI, 1986 ; MENG et al. , 1986) indiquent des taux de recapture ou de survie de 2 a $80 \%$ dans le cas de préestivaux ou d'estivaux de corégones contre seulement 0.1 à $4 \%$ dans le cas des alevins vésiculés (ROTH, 1954 ; EINSELE, 1965 ; WESTMANN et al., 1984 ; LUCZYNSKI, 1986). Dans plusieurs pays, un effort accru a été porté lors des 15 dernières années sur les techniques de production de juvéniles de corégones soit en étangs (HOCHMAN et al., 1974; WESTMAN et al., 1984; CHAMPIGNEULE, 1985), soit en bassins avec du zooplancton vivant (GUNKEL et KAUSCH, 1979 ; STEFFENS, 1979 ; FLÜCHTER, 1980) ou plus récemment de l'aliment sec (DABROWSKI et al., 1984; BERGOT et 
al., 1986 ; CHAMPIGNEULLE et al., 1986), soit en cages immergées éclairées (BRYLINSKI et al., 1979 ; URYN, 1979 ; JAGER et al., 1984 ; ZAUGG et PEDROLI, 1986). La méthode est fondée sur le fait que, de nuit, certains organismes planctoniques sont attirés par une source lumineuse placée à l'intérieur de la cage. La technique permet de concentrer le zooplancton et donc de le rendre facilement disponible pour les larves qui sont par ailleurs protégées des prédateurs. Le but du présent article est de faire. dans le cas du Léman, un premier bilan des principales caractéristiques de la production de préestivaux de corégones avec la technique d'élevage en cages. Les résultats des repeublements réalisés seront publiés ultérieurement.

\section{MATERIEL ET MÉTHODES}

\section{Installations}

En 1983, 84 et 85, cinq cages identiques conçues par les services de la D.D.A. de Haute-Savoie ont été immergées au Léman sous 5-7 m d'eau dans un site situé à $50 \mathrm{~m}$ de la rive et par des fonds de

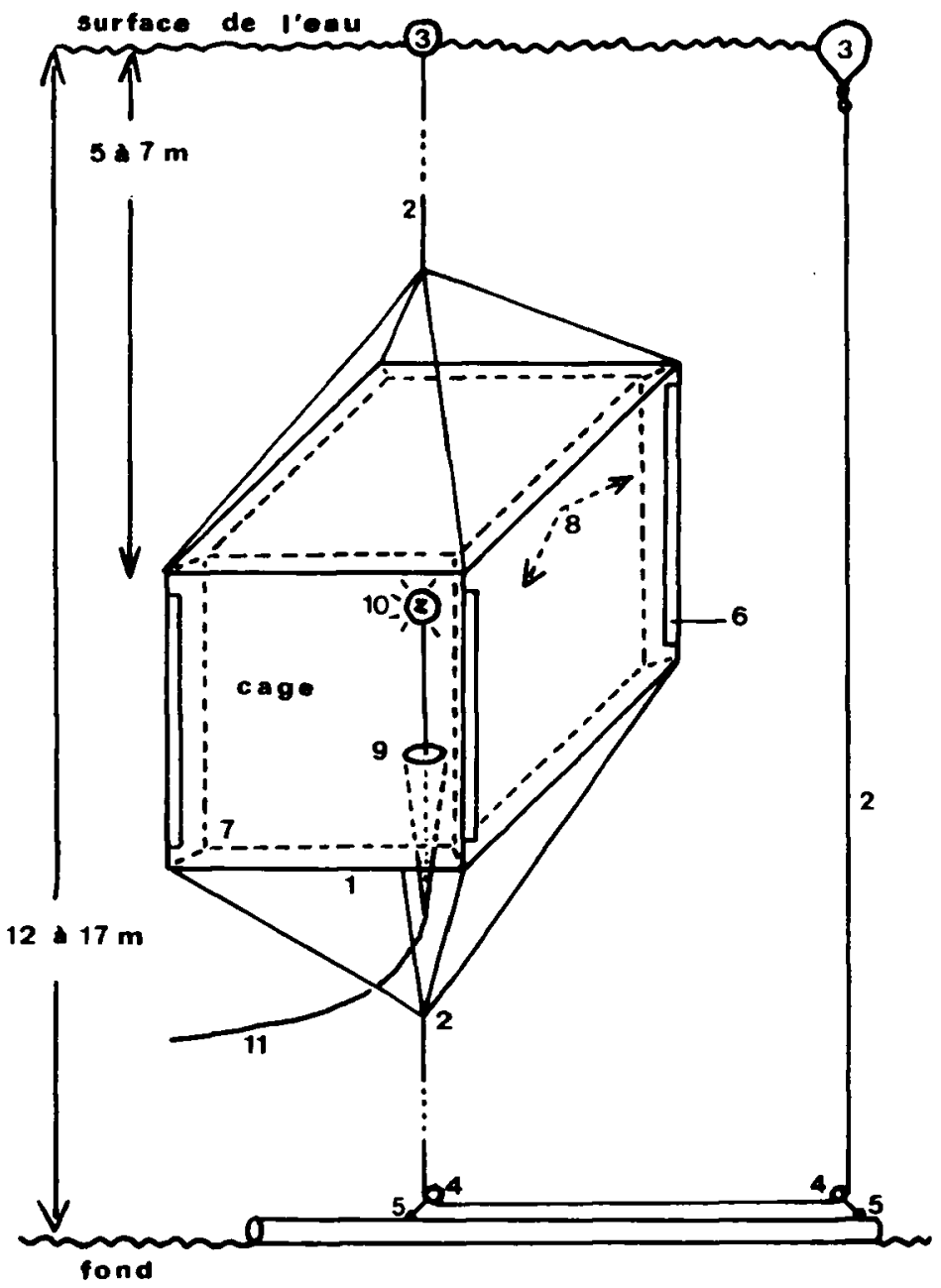

Figure 1: Schéma de montage des cages immergées utilisées au Léman. (d'après CHAMPIGNEULLE et al., 1986).

1 : cadre ; 2 : filins supportant la cage ; 3 : bouées ; 4 : poulies ; 5 : ancrage ; 6 : ballast en tuyau de PVC ; 7 : filet ; 8 : système d'ouverture (fermeture éclair); 9 : système de vidange: 10 : ampoule (100 W, $24 \mathrm{~V}$ ) incluse dans un bocal étanche; 11 : câble électrique (24V).

Figure 1 : Construction schema of a submerged illuminated cage (from CHAMPIGNEULLE et al. , 1986).

1 : frame ; 2 : lines supporting the cage : 3 : buoys ; 4 : blocks ; 5 : anchoring ; 6 : ballast in PVC pipe: 7 : cage net wall; 8 : opening system (zip fastener); 9 : emptying system : 10 : electric bulb $(100 \mathrm{~W}, 24 \mathrm{~V})$ in a water-tight glass-bottle ; 11 : electric cable (24V). 
12 à 17 mètres. La figure 1 précise le schéma de montage des cages. Chacune d'entre elles est constituée d'un cadre rigide assurant la flottaison et le soutien d'une poche cubique (côté : 1.8 m; volume: $5,8 \mathrm{~m}^{3}$ ) en filet nylon de $0,9 \mathrm{~mm}$ de côté de maille. L'éclairage nocturne est réalisé dans chaque cage par une ampoule électrique $(100 \mathrm{~W}, 24 \mathrm{~V})$ placée dans un bocal étanche (type "conserves"). La desserte des cages et les manipulations ont été assurées grâce à l'utilisation d"une barge motorisée munie d'une potence et d'un treuil (photo 1). Les cages ont été remontées et émergées partiellement une fois par semaine afin de procéder à un nettoyage de la poche grâce à une pompe fournissant un jet d'eau orienté de l'intérieur vers l'extérieur.

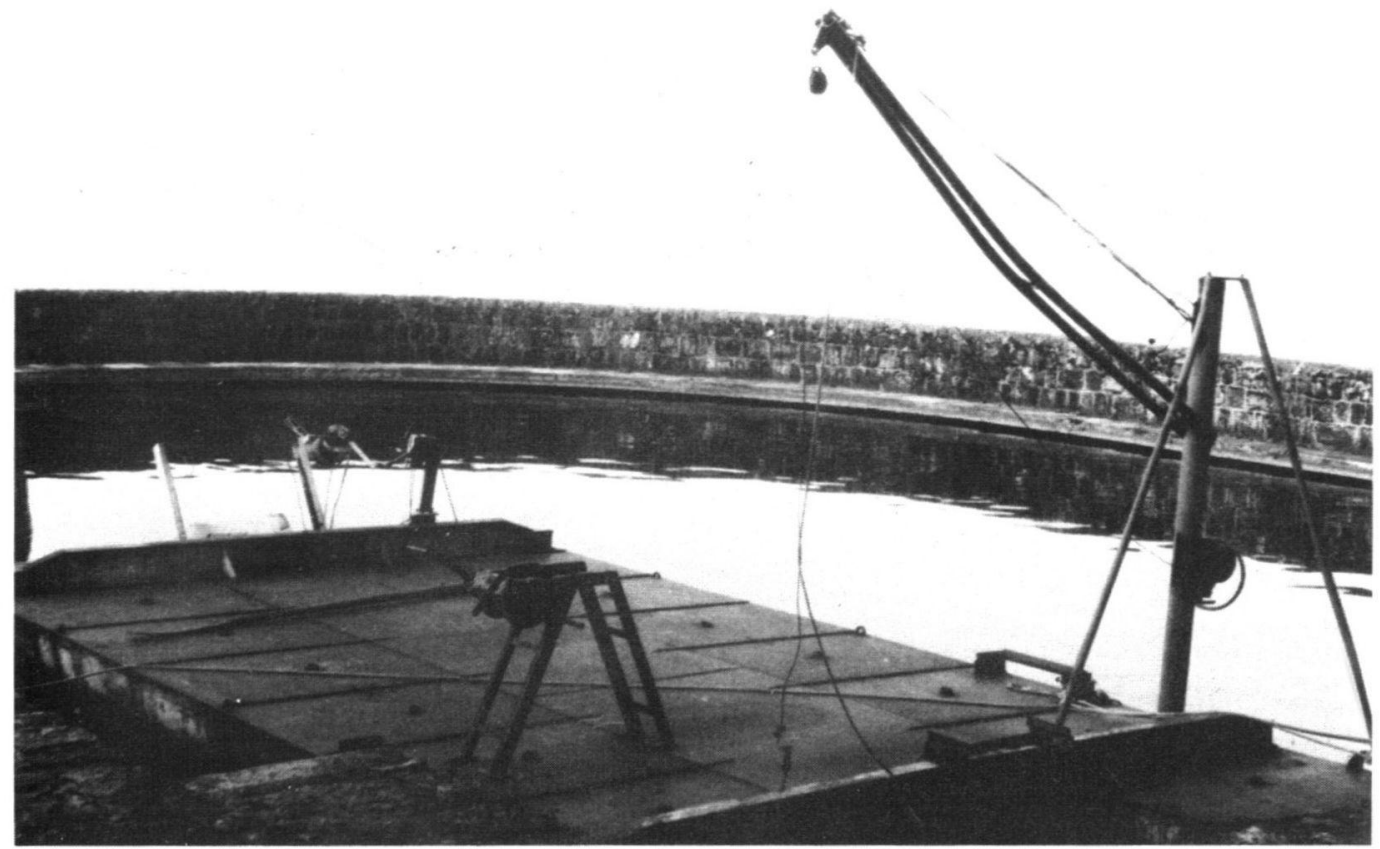

Photo 1: Système de manipulation des cages (barge, potentê et treuil).

Photo 1: Handling system to control cages (barge, hand-winch).

\section{Origine des larves}

Les alevins vésiculés utilisés au cours des 3 années d'expériences proviennent de corégones adultes capturés en période de reproduction, en décembre, avec des filets maillants (45 $\mathrm{mm}$ ) placés à une profondeur de 5 à $30 \mathrm{~m}$ sur la beine de la partie française du Léman. Selon CHAMPIGNEULLE et al. (1983) et GERDEAUX (1984) les reproducteurs sont principalement âgés de $1^{+}$à $3^{+}$. La fécondité des femelles variait de 35 à 55.000 ovules/ $\mathrm{kg}$ (GILLET comm. pers.). Les œufs ont été incubés en bouteilles de Zoug à la pisciculture de la D.D.A. de Haute-Savoie à Thonon avec une eau (pompée en bordure du Léman, à $25 \mathrm{~m}$ de profondeur) dont la température variait entre 5 et $8^{\circ} \mathrm{C}$. En 1985 une partie des œufs utilisés pour l'élevage en cage a été incubée à partir du stade œillé dans une eau refroidie à $2^{\circ} \mathrm{C}$ ce qui a conduit à retarder leur éclosion de 3 semaines. Les alevins ont été conservés en écloserie sans nourrissage, jusqu'à ce que leur vésicule soit à demi résorbée (al. vés. dans la suite du texte).

\section{Mise en charge et récolte}

La mise en charge a eu lieu le 7 mars en 1983 et le 13 février en 1984. Les densités testées étaient de 25.000 al. vés. /cage ( 2 réplicats), 50.000 al. Vés. (2 réplicats) et 100.000 al. vés. /cage soit respectivement 4287,8573 et $17147 \mathrm{ind} / \mathrm{m}^{3}$. Le comptage final du nombre de préestivaux a eu lieu le 14 juin en 1983 (100 jours) et le 5 juin en 1984 (113 jours). En 1983, en milieu de période d'élevage (51 jours), le nombre de juvéniles par cage a été estimé en utilisant la méthode de Petersen (capturemarquage et recapture). Le marquage a été réalisé par coloration des larves grâce à un bain de $30 \mathrm{mn}$ dans une solution (1/25.000) de brun-bismark (BOUTRY, 1983).

L'analyse des résultats de 1983-84 a conduit à réaliser en 1985 un protocole différent visant à préciser l'influence d'une mise en charge soit à partir d'alevins vésiculés retardés de 3 semaines par incubation en eau froide, soit à partir d'alevins prégrossis 3 semaines en bassins avec du zooplancton vivant. Pour toutes les cages, la densité était de 50.000 larves/cage. Les cages témoins (2 réplicats) ont été mises en charge le 13 mars 1985. L'immersion des alevins vésiculés retardés ( 2 cages) et des alevins prégrossis en bassin (1 cage) a eu lieu le 3 avril. Au moment de la mise en charge, le poids (poids 
frais moyen calculé à partir d'un lot de 100 individus égouttés) des alevins prégrossis était de $7 \mathrm{mg}$ contre $5 \mathrm{mg}$ pour les alevins vésiculés. La récolte a eu lieu de 12 juin 1985 soit après 91 jours d'élevage pour les lots témoins et les alevins prégrossis et 70 jours pour les lots retardés.

\section{Suivi de la production}

Le nombre d'alevins déversés par cage a été évalué de la façon suivante: réalisation d'une solution concentrée de larves homogénéisée par brassage, calcul de la densité de larves dans la solution et prélèvement du volume de solution correspondant au nombre de larves désiré. La croissance a été suivie en mesurant la longueur totale et le poids dans des échantillons de 30 à 50 individus par cage prélevés plusieurs fois au cours de la période d'élevage. Pour limiter les biais d'échantillonnage, les prélèvements, réalisés à l'épuisette, ont été faits après avoir concentré les alevins en émergeant les cages jusqu'à ce qu'il reste environ $10 \mathrm{~cm}$ d'eau. Au moment de la récolte le nombre de préestivaux a été compté exactement et un échantilion de 50 à 200 individus a été collecté dans chaque cage pour les mesures de taille et de poids.

\section{Suivi de la température et du zooplancton}

Les caractéristiques thermiques du lac au cours des 3 périodes d'élevage ont été suivies par un enregistrement de la température au niveau de la beine, à $5 \mathrm{~m}$ sous la surface de l'eau. L'évolution relative de l'abondance du zooplancton a été précisée grâce au suivi (BALVAY, comm. pers.) d'un indice évalué comme suit : volume de zooplancton sédimenté évalué à partir de traits verticaux (- 50 à $0 \mathrm{~m})$ réalisés de façon standard en un point situé dans la baie de Thonon en zone pélagique par $150 \mathrm{~m}$ de fond. Le filet à plancton utilisé a un maillage de 200 microns et une ouverture circulaire de 35 centimètres.

\section{RÉSULTATS}

\section{Température et zooplancton}

La température en début d'élevage (fig. $2 \mathrm{~A})$ est restée basse $\left(5 \leqslant T \leqslant 8^{\circ} \mathrm{C}\right)$ pendant plus longtemps en 1984 (73j.) qu'en 1985 (49j.) et 1983 (40j.). En 1985 les alevins retardés n'ont été soumis à une faible température $\left(\leqslant 8^{\circ} \mathrm{C}\right)$ que pendant 28 jours. La mise en charge des cages est réalisée bien avant le pic printanier de zooplancton (volume sédimenté : 50 à $80 \mathrm{ml}$ ) qui a lieu courant juin, plus ou moins tardivement selon l'année (fig. 2 B). Le zooplancton est resté à un niveau bas (volume sédimenté $\leqslant 20 \mathrm{ml}$ ) pendant toute la durée de l'élevage en 1984, pendant environ 11 semaines en 1983 et 1985 (alevins précoces) et pendant seulement 8 semaines en 1985 pour les alevins à mise en charge retardée (fig. 2 B). La récolte ayant lieu en début juin et la période de marquage s'étalant sur 2 à 3 semaines les relachers de préestivaux au Léman ont lieu au cours du pic printanier de zooplancton (fig. 2).

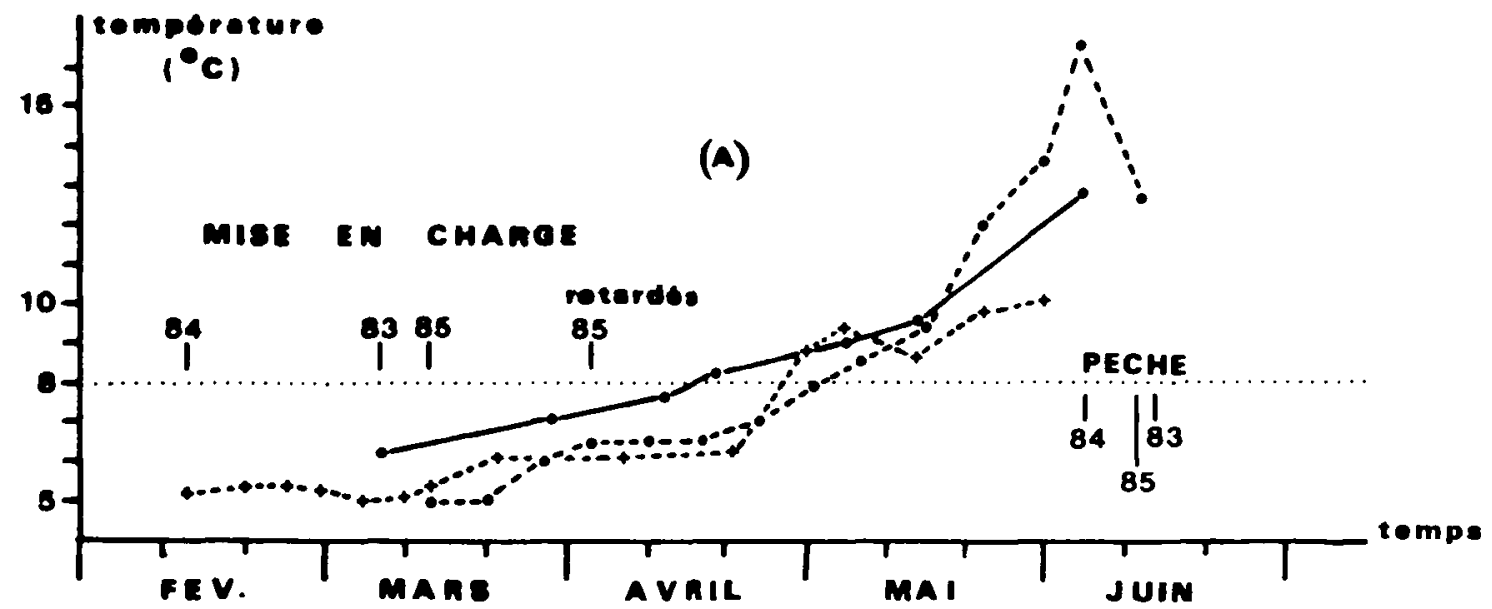

Figure 2: Conditions thermiques et trophiques au cours des essais d'élevage en cages.

2A. Température de l'eau à proximité des cages à $5 \mathbf{m}$ sous la surface.

$(\longmapsto) 1983 ;(+--+) 1984 ;(\cdot--) 1985$.

Figure 2: Thermic and trophic conditions during the cage rearing experiments.

2A. Water temperature near cages at $5 \mathrm{~m}$ under the surface.

$(\ldots+) 1983 ;(+-+) 1984 ;(+\ldots) 1985$. 


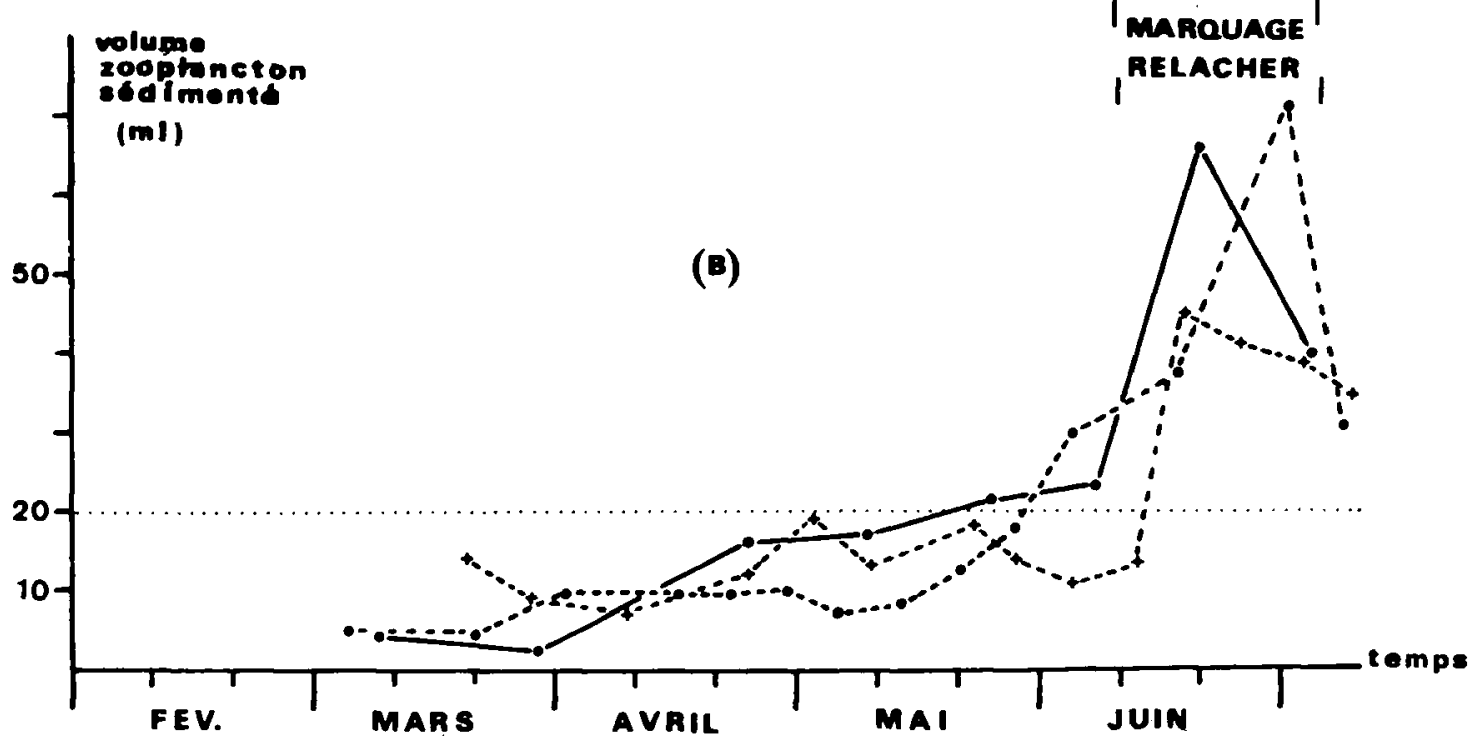

2B. Indice d'évolution du zooplancton (volume de zooplancton sédimenté). $(\longmapsto) 1983 ;(+-+) 1984:(\cdot--\cdot)$ 1985. BALVAY (comm. pers.).

2b. Index of zooplankton evolution (volume of sedimenting zooplankton). $(\longrightarrow) 1983 ;(+--+) 1984 ;(--\longrightarrow+1985$. BALVAY (comm. pers.).

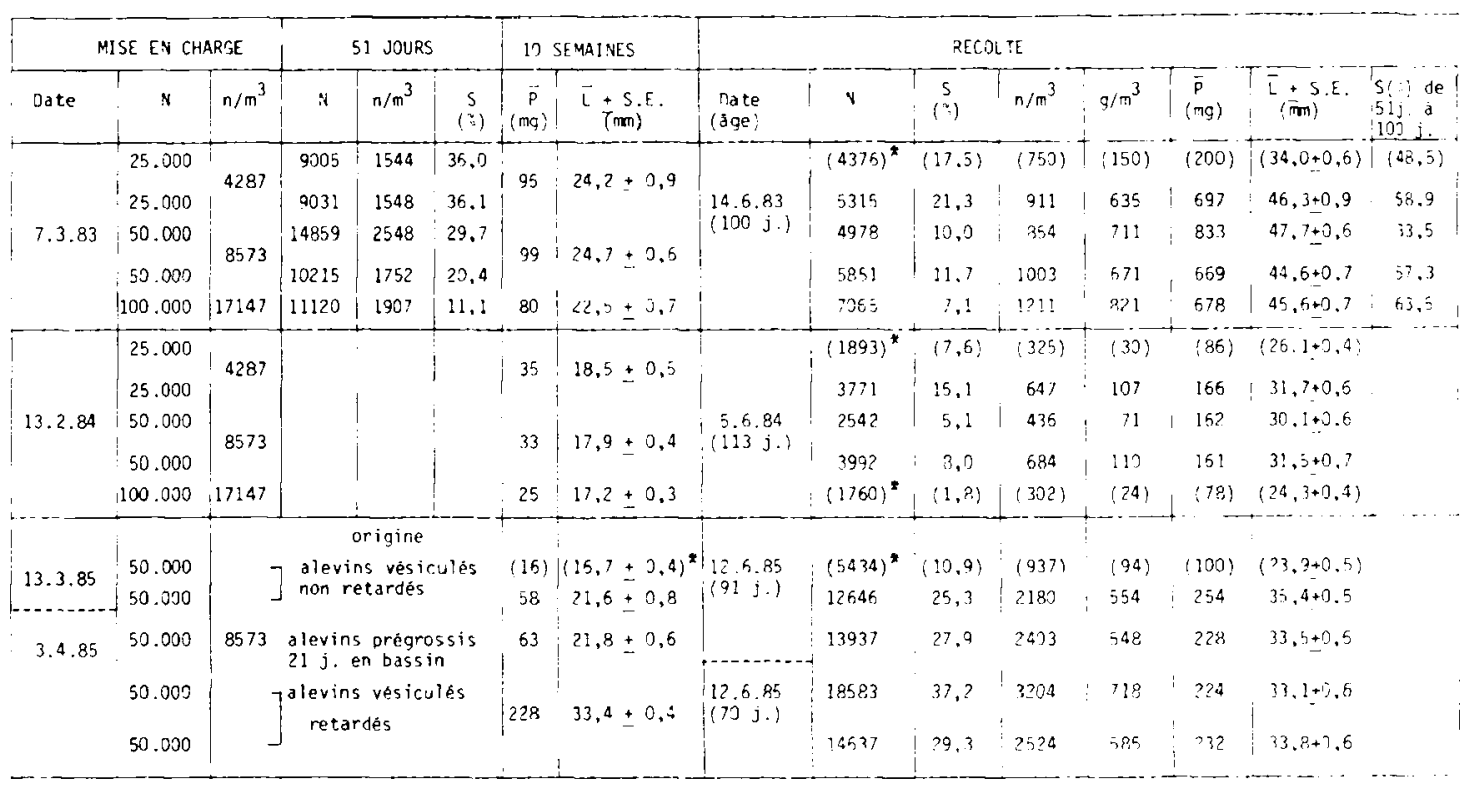

Tableau I: Résultats des élevages de préestivaux de corégones en cages éclairées immergées dans le Léman de 1983 a 1985.

$\mathbf{N}$ : nombre total par cage : $n / \mathbf{m}^{3}=$ nombre par $\mathbf{m}^{3} ; \mathbf{S}:$ survie en $\% ; \overline{\mathbf{P}}:$ poids moyen en $\mathrm{mg}:\left[ \pm S\right.$.E. : longueur moyenne en $\mathrm{mm}$ pour 50 a 200 individus $\pm S . E$. ; $g / \mathrm{m}^{3}$ : biomasse en $\mathrm{g}$ par $\mathrm{m}^{3}$.

$(\quad)^{*}$ cages ayant eu des problèmes de panne d'éclairage.

Table I: Results or rearing coregonid fry in illu minated cages submerged in Lake Léman from 1983 to 1985 .

$\mathbf{N}$ : total number per cage $; n / \mathbf{m}^{3}=$ number par $\mathbf{m}^{3} ; \mathbf{S}:$ survival in $\% ; \overline{\mathbf{P}}:$ mean weight in $\mathrm{mg}: \overline{\mathrm{L}} \pm$ S.E.: mean length in $\mathrm{mm}$ for 50 to 200 individuals $\pm \mathrm{S} . \mathrm{E}$.: $\mathrm{g} / \mathrm{m}^{3}$ : biomass in $\mathrm{g}$ per $\mathrm{m}^{3}$.

()$^{*}$ cage with problems of black out during experiment. 


\section{Survie (tableau 1, p. 5)}

En 1983 la survie variait selon les cages entre 11,1 et $36,1 \%$ à 51 jours et entre 7,1 et $21,3 \%$ à 100 jours. Pour les 3 mises en charge testées ( 25 - 50 et 100.000 /cage) la survie diminue lorsque la mise en charge augmente. La survie estimée en 1983 entre le 51 ème et le 100ème jour était comprise entre 33,5 et $63,5 \%$. Pour chacune des cages la survie au cours de la deuxième moitié de la période d'élevage est nettement supérieure à celle observée au cours de la première moitié (tab. 1). Notons que la plus faible survie en 2 ème période d'élevage a été observée dans la cage ayant la plus forte densité intermédiaire.

En 1984, la survie en fin de période d'élevage (à 113 jours) était nettement plus faible qu'en 1983, variant entre 5,1 et $15,1 \%$.

En 1985, la survie en fin de période d'élevage (à 70 ou 91 jours) variait entre 25,3 et $37,2 \%$. La survie en début juin 1985 était donc nettement supérieure à celle observée en $1983(10,0-11,7 \%)$ et $1984(5,1-8.0 \%$ ) avec la même mise en charge initiale de 50.000 alevins vésiculés par cage). En 1985 la survie observée à la récolte, dans les cages à mise en charge retardée $(29,3$ - 37,2\%), est significativement $(\alpha=1 \%$ ) supérieure à celle observée dans toutes les autres cages.

\section{Récolte (tableau 1)}

Pour l'ensemble des cinq cages regroupées la récolte finale totale était de 27.585 préestivaux en 1983 contre seulement 13.958 en 1984. La biomasse totale récoltée était environ 9 fois plus élevée en $1983(17,43 \mathrm{~kg})$ qu'en $1984(1,98 \mathrm{~kg})$. Le poids moyen individuel final était environ 4,5 fois plus élevé en 1983 (632 mg) qu'en 1984 (142 mg)

En 1983 la récolte finale par cage variait entre 4978 ind. (854 ind $\left./ \mathrm{m}^{3}\right)$ et $7065 \mathrm{ind} .\left(1211 / \mathrm{m}^{3}\right)$ avec une biomasse comprise entre 635 et $821 \mathrm{~g} / \mathrm{m}^{3}$. En 1983 la biomasse récoltée augmente, mais non proportionnellement, lorsque la mise en charge augmente (tab. 1). Le nombre final de préestivaux récoltés est plus élevé dans la cage de plus forte charge initiale (100.000) que dans les autres. A 51 jours le nombre de survivants était plus faible (9005 à 9031 ind./cage) dans les cages de plus faible r.ise en charge (25.000) que dans les autres (10.215 à 14.859 ind./cage). La plus forte densité intermédiaire a été observée dans une cage ayant une charge initiale de 50.000 .

En 1984 la récolte finale par cage variait entre 2542 (436 ind./ $\mathrm{m}^{3}$ ) et 3992 préestivaux $\left(684 / \mathrm{m}^{3}\right)$ pour une biomasse comprise entre 71 et $110 \mathrm{~g} / \mathrm{m}^{3}$. II n'y avait pas de ralation évidente entre la récolte finale et la mise en charge initiale.

En 1985 l'ensemble des cinq cages regroupées a conduit à une récolte finale totale de 65237 préestivaux. La récolte totale ne peut être comparée à celle de 1983 et 1984 puisque le protocole de mise en charge était différent. Cependant les comparaisons sont possibles entre les cages de même mise en charge initiale (50.000). En 1985 la récolte finale par cage variant entre $12646\left(2180 \mathrm{ind} . / \mathrm{m}^{3}\right)$ et 18583 préestivaux ( $3204 \mathrm{ind} . / \mathrm{m}^{3}$ ) est nettement plus élevée que celle observée à la même époque (début juin) dans les cages à 50.000 lors des deux années précédentes (tab. 1). Par ailleurs, malgré une durée d'élevage plus courte (70 ou 91 jours) la biomasse récoltée (554 à $718 \mathrm{~g} / \mathrm{m}^{3}$ ) en 1985 est nettement plus élevée qu'en $1984\left(71\right.$ à $110 \mathrm{~g} / \mathrm{m}^{3}$ pour 113 jours) et voisine de celle observée en 1983 (671 à $711 \mathrm{~g} / \mathrm{m}^{3}$ pour 100 jours).

\begin{tabular}{|l|c|c|c|c|}
\hline Année ou lot & 1984 & $\begin{array}{c}1985 \\
\text { non retardés }\end{array}$ & 1983 & $\begin{array}{c}1985 \\
\text { retardés }\end{array}$ \\
\hline $\begin{array}{l}\text { Longueur moyenne } \\
(\mathrm{mm}) \pm \mathrm{S} . \mathrm{E} . \text { (n }>100)\end{array}$ & $17,9 \pm 0,4$ & $21,6 \pm 0,8$ & $24,7 \pm 0,6$ & $33,4 \pm 0,4$ \\
\hline $\begin{array}{l}\text { Nombre de jours où } 1 \mathrm{a} \\
\text { température a été } \leqslant 8^{\circ} \mathrm{C}\end{array}$ & $\begin{array}{c}10 \text { semai- } \\
\text { nes }\end{array}$ & 49 jours & 40 & 28 \\
\hline
\end{tabular}

Tableau II: Longueur moyenne à 10 semaines pour les corégones élevés en cage (mise en charge: 50.000 alevins vésiculés) selon l'année ou le lot et le nombre de jours d'élevage avec une température de l'eau $\leqslant 8^{\circ} \mathrm{C}$.

Table II: Average length of coregonid fry after 10 weeks in cages (initial stocking: 50.000 sac fry/cage) according to the year or the batch and the number of rearing days with a water temperature $\leqslant 8^{\circ} \mathrm{C}$. 


\section{Croissance}

Les courbes (fig. 3 ) indiquent une faible croissance in itiale. En effet, le poids de $50 \mathrm{mg}$ (longueur $18-20 \mathrm{~mm}$ ) est atteint en 50 à 70 jours (fig. 3). Ensuite le poids a rapidement augmenté en 1983 et 1985 mais non en 1984.

Pour une année donnée (1983 ou 1984) la longueur moyenne à 10 semaines n'est pas significativement ( $\alpha=5 \%$ ) différente entre les cages initialement chargées à 25.000 ou 50.000 alevins vésiculés. Cependant la longueur moyenne à 10 semaines est significativement l $\alpha=1 \%$ en 1983 et $\alpha=5 \%$ en 1984) inférieure dans la cage à 100.000 comparitivement aux cages ayant reçu une mise en charge inférieure (tab. 1). La différence est encore significative après 91 jours d'élevage en $1984(21,1 \mathrm{~mm} / 23,6-24,6 \mathrm{~mm}$ ) mais elle ne subsiste plus après 100 jours en 1983 . Pour une mise en charge donnée la longue ur moyenne à 10 semaines est significativement ( $\alpha=1 \%$ ) inférieure en 1984 comparativement à 1983 (tab. 1). En 1985, avec une mise en charge identique de 50.000, la taille moyenne à 10 semaines dans les cages à alevins vésiculés retardés $(33.4 \mathrm{~mm})$ est significativement $(\alpha=1 \%$ ) plus élevée que celle observée dans les cages à alevins plus précoces $(21,6 \mathrm{~mm})$ ou prégrossis. Le tableau II met en évidence que, avec une mise en charge initiale de 50.000 alevins vésiculés, la taille moyenne à 10 semaines est d'autant plus élevée que le nombre de jours où la température de l'eau est $\cong 8^{\circ} \mathrm{C}$ est faible.

La longueur et le poids moyen final à la récolte en début juin varient moins fortement d'une cage à l'autre pour une année donnée comparativement aux variations observées d'une année à l'autre

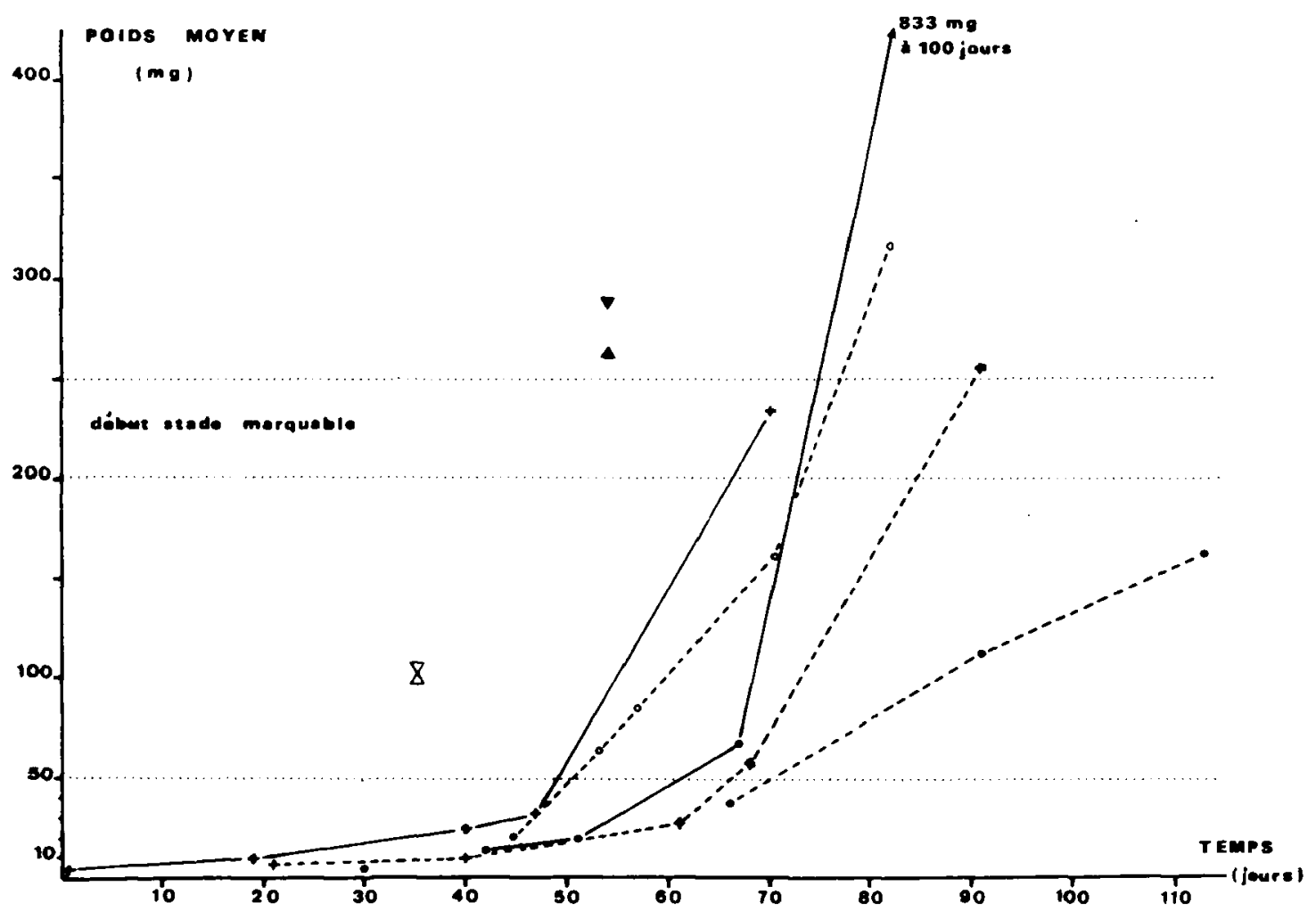

Figure 3: Evolution du poids corporel des alevins de corégones originaires du Léman et élevés en cages (mise en charge 50.000) dans le Léman de 1983 à 1985 . (־) 1983 : $(\bullet--\bullet) 1984 ;(+--+) 1985$ normaux, $(+-+) 1985$ retardés.

Comparaison d̀ d'autres essais :

(_- - - $)$ C. peled en cages au Léman en 1982

(V) DABROWSKI et al., 1984 et (7) BERGOT et al. (1986): corégones du Léman élevés en bassins $\left(14-15^{\circ} \mathrm{C}\right)$ avec un aliment sec à base de levure.

Figure 3: Body weight of alevins from Lake Léman coregonids reared in cages (stocking:

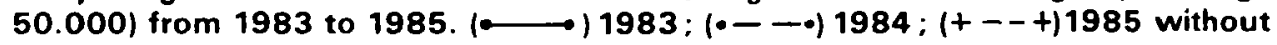
delayed hatching: $(+-+) 1985$ with delayed hatching.

Comparison with other experiments:

(.- - - $)_{0}$ C. peled in Lake Léman cages in 1982

(V) DABROWSKI et al. 1984 and (V) BERGOT et al. (1986) : coregonids from Lake Léman reared in tanks and fed with a dry food with yeast (temperature $14-15^{\circ} \mathrm{C}$ ). 
(tab. 1). En 1984, malgré une durée d'élevage plus longue (113 jours) la taille moyenne dans les cages à 50.000 est significativement $(\alpha=1 \%$ ) inférieure $(30,1-31,5 \mathrm{~mm})$ à celle observée en $1983(44,6$ $47.7 \mathrm{~mm}$ pour 100 jours) et en 1985 (33,1 - 35.4 mm pour 70 à 91 jours). En 1985 la taille à la récolte dans le cas des alevins retardés est voisine $(33,1-33,8 / 33,5-35,4 \mathrm{~mm})$ de celle des alevins précoces ou prégrossis et ce malgré une durée d'élevage inférieure de 3 semaines. Mis à part le cas de 1984 le stade "préestivaux marquables" (200 à $250 \mathrm{mg}$ selon CHAMPIGNEULLE et ESCOMEL, 1984) par cautérisation de l'adipeuse est atteint en 65 à 90 jours (fig. 3).

\begin{tabular}{|c|c|c|c|c|c|c|c|}
\hline \multicolumn{3}{|c|}{ Mise en charge } & \multicolumn{5}{|c|}{ Récol te } \\
\hline Espèces & Année & $\begin{array}{l}\text { Nore par } \\
\text { cage }\end{array}$ & $\begin{array}{l}\text { Date } \\
\text { (âge) }\end{array}$ & Nombre & $\begin{array}{c}S \\
(\%)\end{array}$ & $\begin{array}{c}\bar{p} \\
(m g)\end{array}$ & $\bar{L}+\underset{(m m)}{S . E .}$ \\
\hline Coregonus peled & 1982 & 100.000 & $\begin{array}{l}5-7-82 \\
(82 \text { jours })\end{array}$ & 6425 & 6,4 & 316 & $37,2 \pm 3,6$ \\
\hline Esox lucius & 1983 & 50.000 & $7-6-83$ & 1765 & 3,5 & - & $30-40$ \\
\hline Salmo trutta & 1985 & 2.000 & $12-6-85$ & 1530 & 76,5 & 740 & $45,0 \pm 1,1$ \\
\hline
\end{tabular}

Tableau III: Résultats de quelques essais complémentaires d'élevage en cages éclairées immergées dans le Léman.

$S$ : survie en $\% ; \overline{\mathbf{P}}$ : poids moyen en $\mathbf{m g}$ : $\bar{L}$ : longueur moyenne en $\mathbf{m m}$.

Table III : Results of some additional experiments with illuminated cages submerged in Lake Léman.

$S:$ survival in $\%: \overline{\mathbf{P}}$ : mean weight in $\mathbf{m g} ; \bar{L}:$ mean length in $\mathrm{mm}$.

\section{Essais d'élevage en cages avec d'autres espèces}

Sur le même site du Léman et avec la même technique, des essais très limités ont été pratiqués à partir d'alevins vésiculés de truite (Salmo trutta) issus de géniteurs du Léman, de brochet (Esox lucius) et de corégone peled (Coregonus peled) d'origine tchèque. Le tableau II résume les résultats obtenus. En ce qui concerne le peled la croissance observée en 1982 avec une mise en charge tardive (début avril) est bonne et voisine des meilleures croissances observées avec le corégone du Léman (fig. 3)

\section{DISCUSSION}

Pour les juvéniles de poissons zooplanctonophages, la technique d'élevage en cages éclairées permet de s'affranchir des problèmes de collecte rencontrés lors des élevages en bassin avec apport de zooplancton vivant (CHAMPIGNEULLE et al., 1986). La technique, surtout utilisée dans le cas des corégones, a également été employée avec succès dans le cas d'autres espèces : sandre (Stizostedion lucioperca) et tanche (Tinca tinca) selon MAMCARZ et SZCZERBOWSKI (1984), brochet (Esox /ucius) d'après JÄGER (1986) et ZAUGG et PEDROLI (1986) et truite /Salmo trutta) lacustre (ZAUGG et PEDROLI, 1986). Les installations d'élevage employées dans le Léman se sont montrées bien adaptées au manque de zones abritées. Néanmoins le système est plus coûteux et difficile à contrôler que lorsque les cages peuvent être fixées à un ponton accessible à pied depuis la rive. Un prototype de cage sur pieds reposant sur le fond, conçu dans le cadre de la présente étude, a été testé en 1984 et 85 dans le Léman ; il permet de s'affranchir des installations d'ancrage et du système de poulies.

Les premiers résultats obtenus de 1983 à 1985 montrent que, dans les conditions de l'élevage en cage pratiqué au Léman, il y a de fortes variations interannuelles dans la croissance et la survie des corégones jusqu'au stade "préestivaux". La mise en charge précoce et la faible température en début d'élevage semblent pouvoir en partie expliquer les mauvais résultats obtenus en 1984. Les essais de 1985 renforcent cette hypothèse puisque le fait de différer de seulement 3 semaines la mise en charge a permis d'améliorer la croissance et la survie des larves. La température semble être un facteur initial important puisque, à charge égale, la croissance au cours des deux premiers mois a été d'autant meilleure que la température a été plus élevée. Selon Mc CORNICK et al. (1971) la croissance de Coregonus artedi au cours du premier mois augmente quand la température augmente entre 3 et $18^{\circ} \mathrm{C}$. GERDEAUX et DEWAELE (1986) ont mis en évidence l'existence d'une corrélation positive entre 
le tonnage de corégones pêchés dans le Léman et le nombre de degrés-jour (au-delà de $8^{\circ} \mathrm{C}$ ) lors de l'année correspondant à la naissance des corégones pêchés. Les fortes variations interannuelles ne sont pas spécifiques au cas du Léman. En effet MAMCARZ et SZCZERBOWSKI(1984) ont montré dans le cas d'un lac polonais que la survie à 2 mois de préestivaux élevés en cages varie fortement d'une année à l'autre : entre 3.1 et $72,6 \%$ pour Coregonus lavaretus. Les auteurs indiquent également que, selon le type de lac. la biomasse de préestivaux varie de 0.1 à $2.8 \mathrm{~kg} / \mathrm{m}^{3}$. Les résultats obtenus au Léman $\left(0,1\right.$ à $\left.0,8 \mathrm{~kg} / \mathrm{m}^{3}\right)$ se situent dans la partie inférieure de cette gamme. Dans les essais pratiqués au Léman l'élevage en cage est caractérisé par une survie (11-36\%), une croissance (19-33 mg) et une biomasse (0,2-0,3 kg/cages) relativement faibles en début d'élevage (51 jours en 1983). Dans plusieurs lacs polonais avec Coregonus lavaretus et avec les mises en charges similaires, BRYLINSKI et al. (1979) et URYN (1979) indiquent après 51 -53 jours des survies de 33 à $85 \%$, des poids moyens de 19 à $135 \mathrm{mg}$ et des biomasses de 0,7 à $8,7 \mathrm{~kg} /$ cage. Selon BRYLINSKI et al. (1979) les meilleurs résultats ont été obtenus dans de petits lacs, riches en zooplancton et s'échauffant rapidement au printemps. Ces données suggèrent la nécessité d'associer les deux facteurs : température et zooplancton (quantité et qualité). D'après DABROWSKI(1983) les nauplii de copépodes de 0,2-0,25 mm représentent le type d'aliment le plus fréquemment rencontré dans les contenus stomacaux de larves de corégones en milieu naturel. Dans le Léman, selon BALVAY (comm. pers.) le pic printanier de zooplancton a lieu en fin mai-juin à une période variable selon l'année et généralement après que l'isotherme $10^{\circ} \mathrm{C}$ ait atteint la profondeur de $10 \mathrm{~m}$. Par conséquent, une mise en charge tardive est susceptible de diminuer les risques liés à la faible abondance du zooplancton. En 1984, année où la croissance a été très faible. plusieurs facteurs défavorables ont été cumulés: mise en charge précoce, température basse et amorce tardive du pic printanier de zooplancton. Le suivi des essais de 1983 a montré que la mortalité est nettement plus forte au cours de la première moitié de la période d'élevage qu'au cours de la seconde. Par ailleurs la survie et la croissance en début d'élevage sont inférieures dans les cages ayant eu la charge initiale la plus forte (100.000 alevins vésiculés/cage). Ces différences pourraient être en partie liées à la quantité de plancton en début d'élevage qui ne serait pas disponible en quantité suffisante vis-à-vis des exigences des larves. En effet GALLIOT et MAUFFOY (1983) ont examiné les contenus stomacaux des larves âgées de 21 jours issues des cages du Léman. L'étude a montré la présence d'un fort pourcentage de larves ayant l'estomac vide, ce pourcentage étant plus élevé 150 à $100 \%$ des échantillons prélevés) dans les cages initialement chargées à 100.000 alevins que celui (20 à $30 \%$ ) observé dans les cages moins chargées. DABROWSKI et al. ont cependant montré que la survie au jeûne est relativement élevée dans le cas des corégones du Léman puisque, à $15^{\circ} \mathrm{C}$, la mortalité totale (100\%) n'est atteinte qu'au 24ème jour après l'éclosion. Parallèlement aux élevages en cages, des larves de corégones ont été élevées en bassin avec du zooplancton capturé dans le Léman (CHAMPIGNEULLE et al., 1986). Avec une distribution de plancton en excès et une température voisine de celle de l'élevage en cage, la survie jusqu'à 50 jours en bassin est supérieure à celle observée en cage ce qui renforce l'hypothèse d'un rapport densité en zooplancton/densité en larves trop faible en début d'élevage. DABROWSKI (1976) indique que, pour les larves de Coregonus lavaretus, la densité minimale de zooplancton (individus de $0,004 \mathrm{mg}$ ) permettant une bonne croissance est de 50-70 ind/I, et que l'optimum est atteint à partir de 200-260 ind./I. Notons que des concentrations de perches et de gardons ont été observées autour des cages et que SZCZERBOWSKI et MAMCARZ (1984) ont montré dans un lac polonais qu'il y a une compétition possible vis-à-vis du plancton attiré. Notons enfin qu'il peut y avoir dans certains cas de fortes mortalités en élevage en liaison avec la mauvaise qualité du plancton (ECKMANN et al., 1986) ou avec des problèmes de parasitisme (BATURO, comm. pers.). Par contre le milieu "cage" constitue une protection contre la prédation et, généralement, aucun développement de prédateurs n'est observé à l'intérieur des cages.

Les essais de 1985 ont montré que la technique d'incubation en eau froide, récemment affinée par LUCZINSKI (1984), permet d'améliorer les performances d'élevage en rendant possible une mise en charge retardée. Le décalage de la mise en charge peut être également facilité en utilisant les œufs issus soit de femelles sauvages à ponte tardive, soit de femelles d'élevage dont la ponte est artificiellement retardée par contrôle de la photopériode et de la température (technique utilisée au Japon, DABROWSKI, comm. pers.). Les résultats sont également susceptibles d'être améliorés en limitant les problèmes (mauvaise circulation de l'eau et du zooplancton, accu mulation des fèces) liés au colmatage du filet. En effet, en 1983, la croissance obtenue dans les cages du Léman est similaire (environ $45 \mathrm{~mm}$ après $100 \mathrm{j}$ ) à celle obtenue dans des conditions voisines (sans changement de maille, même espèce. même année) au lac de Neuchâtel (Suisse) par ZAUGG et PEDROLI(1986). Or ces derniers auteurs ont mis en évidence une augmentation de la croissance (environ $50 \mathrm{~mm}$ à 100j) lorsque le maillage des poches passe de 710 à $1320 \mu$ après le premier mois d'élevage. La technique d'accroissement du maillage des filets au cours de la croissance est pratique courante dans l'élevage en cages des corégones (URYN, 1979 ; BAYLINSKI et al. , 1979 ; JÄGER et al., 1984 ; MAMCARZ et SZCZERBOWSKI, 1984).

JÄGER (1986) a évalué à environ 25 à 53 marks allemands (DM) le coût de production (salaires exclus) de 1000 corégones de $7 \mathrm{~cm}$ produits dans le cadre d'une production commerciale de corégones en cages. Un premier calcul sommaire sur la base des données de BOUTRY (1983) montre que le prix de revient (salaires compris et représentant $35 \%$ du coût) des préestivaux de corégone a varié selon 
l'année au Léman entre 0,4 et $1.8 \mathrm{~F}$ pièce. Cette première évaluation, tant que le taux de recapture n'est pas connu, incite à la prudence quant à l'utilisation de la technique des cages pour la production à grande échelle de corégones de repeuplement. Par ailleurs il importe, dans le contexte des lacs subalpins, de prendre en compte les possibilités offertes par d'autres techniques de production. En effet des études récentes (DABROWSKI et al., 1984 ; BERGOT et al., 1986 ; CHAMPIGNEULLE et al., 1986) ont démontré la faisabilité de l'élevage de juvéniles de corégones du Léman en bassins avec l'utilisation d'un aliment $\mathrm{sec}$ à base de levures fabriqué à la Station INRA de St-Pée-sur-Nivelle. En conditions optimisées (nettoyages par transvasement fréquents, température : $14-16^{\circ} \mathrm{C}$, aliment expérimental distribué a utomatiquement à forte fréquence) et à petite échelle, les performances obtenues sont nettement supérieures à celles réalisées en milieu naturel avec la technique des cages (fig. 3). En effet DABROWSKI et al. (1984) indiquent un poids de 98 à $107 \mathrm{mg}$ obtenu en 35 jours avec une survie de $81-92 \%$ dans une eau à $15^{\circ} \mathrm{C}$. BERGOT et al. (1986) ont obtenu un poids de $260-290 \mathrm{mg}$ en 54 jours sur une survie de $88.92 \%$ dans une eau à $14^{\circ} \mathrm{C}$. Avant de pouvoir conclure il reste à expérimenter la technique dans le cadre d'un programme pilote (x 10.000) permettant de tester les problèmes de changement d'échelle et de faire une évaluation économique. Il faut également évaluer la survie en milieu naturel des préestivaux nourris sur aliment inerte comparativement à celle des préestivaux ayant consommé du plancton vivant. Notons enfin que des essais récents (INRA Thonon) indiquent la possibilité d'obtenir des géniteurs avec alimentation sèche alors que selon MAMCARZ et SZCZERBOWSKI (1984) le stade géniteurs matures n'est pas atteint en cages.

\section{CONCLUSION}

L'étude a montré la faisabilité de la production de préestivaux de corégones en cages immergées éclairées dans les conditions du Léman. Les données acquises sur le Léman et dans d'autres lacs permettent d'aboutir aux recommandations suivantes:

- lorsque la température est trop froide au démarrage (cas du Léman). différer la mise en charge en utilisant soit des alevins vésiculés issus d'cufs à éclosion tardive (incubation en eau froide et/ou récoltes de pontes tardives), soit des alevins prégrossis:

- accroître le maillage des poches au fur et à mesure de la croissance:

- l'élevage en cages ne devrait être pratiqué que dans les cas où les conditions locales sont optimales pour l'utilisation de la technique. Les lacs abrités, petits, riches en zooplancton et chauffant vite en fin d'hiver début de printemps semblent plus favorables si l'on se réfère aux études polonaises:

- réaliser des essais préalables pendant plusieurs années afin d'optimiser la mise en charge (période, densité initiale) et d'évaluer les fluctuations interannuelles des performances zootechniques et économiques:

- il apparaît raisonnable, avant de multiplier les essais d'élevage de corégones en cage, d'attendre les résultats obtenus dans le cadre d'un programme pilote réalisé à l'INRA de St-Pée-sur-Nivelle et de Thonon avec la technique d'élevage en bassins et une alimentation sèche bien adaptée.

Pour optimiser les programmes de pacage lacustre avec des juvéniles de corégones dans les lacs et grandes retenues d'eau, les programmes de recherche doivent se poursuivre dans les domaines suivants:

- performances biotechniques et coûts de production des juvéniles jusqu'à divers stades (vésiculés, alevins de $2 \mathrm{~cm}$, préestivaux et estivaux) selon les différentes techniques de production (en bassins avec de l'aliment inerte et en cages avec du plancton vivant);

- grilles de mises en charge, taux de survie et/ou de recapture selon la taille, la période et l'alimentation (zooplancton/aliment sec) des juvéniles produits et les caractéristiques du plan d'eau (milieu, peuplement en place):

- appréciation du succès de la reproduction naturelle et importance des facteurs limitant (qualité de l'eau, compétition, prédation et pêche).

Les recherches définies ci-dessus sont actuellement menées par l'INRA à la Station d'Hydrobiologie Lacustre de Thonon.

\section{REMERCIEMENTS}

Nous remercions vivement les services de la D.D.A. de Haute-Savoie qui ont mis en place et géré sous l'aspect technique les installations du programme pilote d'élevage de corégones en cages tout en facilitant le suivi scientifique. 


\section{BIBLIOGRAPHIE}

BATIAS A., 1954. Le lavaret du Bourget : productivité et alevinage in Colloque d'économie piscicole des lacs à corégones, 2-15. Station de Recherches lacustres, Thonon.

BERGOTP., CHARLON N and DURANTEH., 1986. The effect of compound diets feeding on growth and survival of coregonid larvae. Arch. Hydrobiol., Beih. Ergebn. Limnol., 22, (sous presse).

BOUTRYE., 1983. Les corégones du Léman : pêche, repeuplement et essais d'élevage de juvéniles en cages immergées. Rapp. Inst. Limnol., Thonon, 10, $32 \mathrm{p}$.

BRYLINSKIE., RADZIEJ J. and URYN B., 1979. Rearing of whitefish (Coregonus lavaretus L.) larvae and fry in illuminated cages. Rocz. Nauk. Poln, 99, 55-57 (en polonais).

CHAMPIGNEULLE A., GERDEAUX D. et GILLET C., 1983. Les pêches de géniteurs de corégones dans le Léman français en 1982. Bull. Fr. Piscic., 290, 149-157.

CHAMPIGNEULLE A. et ESCOMEL J., 1984. Note technique. Marquage de salmonidés de petite taille par ablation de l'adipeuse ou des nageoires pelviennes. Bull. Fr. Piscic. 293-294, 52-58.

CHAMPIGNEULLE A., 1985. Analyse bibliographique des problèmes de repeuplement en salmonidés dans les grands plans d'eau in GERDEAUX D. et BILLARD R., Colloque sur la gestion piscicole des lacs et retenues artificielles, 187-218, INRA Publ., Paris.

CHAMPIGNEULLE A., BOUTRY E., DEWAELE P. and MAUFOY C., 1986. Rearing larvae of Lake Léman coregonid fishes in France. Preliminary data on the production in small ponds, illuminated cages and tanks. Arch. Hydrobiol., Beih. Ergebn. Limnol., 22, (sous presse).

CHRISTIE J., 1963. Effects of artificial propagation and the weather on recruitment in the Lake Ontario whitefish fishery. J. Fish. Res. Board Can., 20, 597-646.

DABROWSKIK, 1976. How to calculate the optimal density of food for fish larvae. Env. Biol. Fish., 1. 87-89.

DABROWSKI K., 1983. Elevage des larves de corégones nourries à l'aliment sec et naturel. Synthèse bibliographique récente. Bull. Fr. Piscic., 291, 183-190

DABROWSKI K., CHARLON N., BERGOT P. and KAUSHIK S., 1984. Rearing of coregonid (Coregonus shinzi palea (Cuv. et Val.)) larvae using dry and live food. I. Preliminary date. Aquaculture, 41 11-20.

ECKMANN R., RÖSCH R., ORTLEPP J. and KLEIFELD G., 1986. The impact of zooplankton from Lake Constance on survival of coregonid larvae. Arch. Hydrobiol., Beih. Ergebn. Limnol., 22, (sous presse).

EINSELE W., 1965. Problems of fish larvae survival in nature and the rearing of economically important middle European freshwater fishes. Calif. Coop. Oceanic Fish. Invest. Rep., 10, 24-30.

FLÜCHTER J., 1980. Review of the present knowledge of rearing whitefish (Coregonidae) larvae. Aquaculture, 19, $191-208$.

GALLIOT M.F. et MAUFOY C., 1983. Elevage larvaire de corégones en cages, bacs, étangs. Mémoire Ecole Supérieure Technique de Biologie Appliquée de $53 \mathrm{p}$

GERDEAUXD., 1984. Statistiques des pêches exceptionnelles de corégones dans les eaux françaises du Léman en 1982 et 83. Rapp. Inst. Limnol., Thonon, 16, 12 p.

GERDEAUX D. and DEWAELE P., 1986. Effects of the weather and of artificial propagation on coregonid catches in Lake Geneva. Arch. Hydrobiol., Beih. Ergebn. Limnol., 22, (sous presse).

GUNKEL G. and KAUSH H., 1979. Feeding and growth of fry and fingerlings whitefish (Coregonus fera J.). E.I.F.A.C. Tech. Pap., 35, 163-176.

HOCHMAN L., PENAZ M. and PROKES M., 1974. The volume of milt, quantity and quality of sperm in Coregonus peled (Gmelin, 1788) from pond culture. Zoologicke listy, 23, 367-380.

JÄGER T., NELLEN W. und SELL H., 1984. Beleuchtate Netzgehegeanlagen zur auf zucht von Fischbrut bis zur Setzlingsgrösse Eine Baunonleitung und Aufzuchtbeschreibung. Berichte aus dem Institut für Meereskunde an der Christian - Albrechts - Universität Kiel, 126, 72 p.

JÄGER T., 1986. Economic aspects of a coregonid culture in illuminated net cages. Arch. Hydrobiol., Beih. Ergebn. Limnol., 22, (sous presse).

LUCZYNSKI M., 1984a. Improvement in the efficiency of stocking lakes with larvae of Coregonus albula $L$. by delaying hatching. Aquaculture, 41, 99-111.

LUCZYNSKI M., 1984b. A technique for delaying embryogenesis of vendace (Coregonus albula L.) eggs in order to synchronize mass hatching with optimal conditions for lake stocking. Aquaculture. 41, 113-117.

LUCZYNSKI M., 1986. Review on the biology, exploitation, rearing and management of coregonid fishes in Poland. Arch. Hydrobiol., Beih. Ergebn. Limnol., 22, (sous presse). 
MAMCARZ A. and SZCZERBOWSKI J.A., 1984 Rearing of coregonid fishes (Coregonidae) in illuminated lake cages. I. Growth and survival of Coregonus lavaretus $\mathrm{L}$ and Coregonus peled $\mathrm{Gmel}$. Aquaculture, 40, 135-145.

MC CORNICK J.H., JONES B.R. and SYRETT R.F., 1971. Temperature requirements for growth and survival of larval ciscos (Coregonus artedi). J. Fish Res. Board Can., 28, 924-927.

MENG H.J., MÜLLER R. and GEIGER W., 1986. Growth, mortality and yield of stocked coregonid fingerlings identified by microtags. Arch. Hydrobiol., Beih. Ergebn. Limnol., 22, (sous presse).

MILLER R.B., 1946. Effectiveness of a whitefish hatchery. J. Wildl. Manage., 10. 316-322.

NÜHMANN W., 1972. The Bodensee: effects of exploitation and eutrophication on the salmonid community. J. Fish. Res. Board Can., 29, 833-847.

PRUUKI V., PURSIAINEN M. and WESTMAN K., 1984. A study of the growth and production of the native whitefish (Coregonus muskun Pallas) and the introduced whitefish (Coregonus peled Gmelin) stocked in two small forest lakes in southern Finland. EIFAC.CECPI/T 42 (Supp/. 1). $91-108$

QUARTIER A., 1954. Les corégones du lac de Neuchâtel et leur pêche. in Colloque d'économie piscicole des lacs à corégones, 16-19. Station de Recherches Lacustres, Thonon.

ROTH A., 1954. Mesures destinées à élever le cheptel de corégones dans les lacs du Canton de Berne. in Colloque d'économie piscicole des lacs à corégones, 25-33. Station de Recherches Lacustres, Thonon.

STEFFENS W., 1979. Coregonenzucht in Industrie massige Fischproduktion, 180-189, VEB Deutscher Landwirtschaftsverlag, Berlin

SZCZERBOWSKI J.A. and MAMCARZ A., 1984. Rearing of coregonid fishes (Coregonidae) in illuminated lake cages. II. Environmental conditions during fish rearing. Aquaculture, 40, 147-161.

TODD T.N., 1986. Artificial propagation of Coregonines in the Management of the Laurentian Great Lakes. Arch. Hydrobiol, Beih. Ergebn. Limnol., 22, (sous presse).

URYN B.A., 1979. Farming of juvenile whitefish (Coregonus lavaretus L.) in submerged illuminated cages. European Mariculture Society Spec. Publ., 4, 289-297.

VAN OSTEN J., 1942. Relationship between the planting of fry and production of whitefish in Lake Erié. Trans. Am. Fish. Soc., 71, 118-121.

VOLGIN M.V., 1973. Cultivation of marketable peled (Coregonus peled Gmelin) in Lake Titivo, Novosibirsk Province. J. Ichthyol., 13, 920-925.

WESTMAN K., ESKELINEN U., TUUNAINEN P. and IKONEN E., 1984. A review of fish stockings in Finland. EIFAC/CECPI/T 42 (SUPpl. 1), 252-268

ZAUGG B. and PEDROLI J.C., 1986. The rearing of young fish in lighted cages. Arch. Hydrobiol., Beih. Ergebn. Limnol., 22, (sous presse). 